\title{
21. PALEOMAGNETIC RESULTS FROM BASEMENT ROCKS FROM SITE 917 (EAST GREENLAND MARGIN) ${ }^{1}$
}

\author{
Didier Vandamme ${ }^{2}$ and Jason R. $\mathrm{Ali}^{3}$
}

\begin{abstract}
During Ocean Drilling Program Leg 152, $779 \mathrm{~m}$ of basaltic lavas within the oldest part of the seaward-dipping reflector sequence (SDRS) was drilled in Hole 917A. We present the results of studies of 150 paleomagnetic specimens sampled from 68 igneous units. We tried to reorient basalt pieces using Formation MicroScanner data so that declinations could be determined, but the attempt failed. Using paleointensity experiments, we also tried to test the thermal origin of magnetization of several units that may have recorded a geomagnetic short event. Chemical changes that occurred in minerals in the samples during heating prevented any conclusions from being reached. Conventional paleomagnetic experiments, however, allowed us to determine the inclinations of 74 of the 92 igneous units, directly or indirectly. The reversed polarity of the entire sequence and the presence of two cryptochrons recorded by three (possibly five) units in the Lower Series and one unit in the Middle Series are consistent with an extrusion of the volcanic sequence of Site 917 during the magnetic Chrons 24r, 25r, or 26r, but exclude magnetic Chron 27r.
\end{abstract}

\section{INTRODUCTION}

Ocean Drilling Program (ODP) Leg 152 drilled Hole 917A about $50 \mathrm{~km}$ off the coast of southeastern Greenland. Hole 917A penetrates the entire lava pile, which was formed during the initial stage of separation of Greenland from Europe. This volcanic pile is called a seaward-dipping reflector sequence (SDRS) because of its special structure, imaged by seismic studies (Larsen, Saunders, Clift, et al., 1994). The principal objective of Leg 152 was the detailed study of the SDRS. We present the paleomagnetic results obtained from 74 of the 92 drilled igneous units.

\section{LABORATORY EXPERIMENTS}

In general, one minicore sample was collected from each igneous unit from which oriented pieces were available. Thick flows were multiply sampled to assess the paleomagnetic similarity between successive cores. We also multiply sampled flows $61-65$ to test the primary origin of their magnetization (see below) by several experimental methods.

Sixty-five igneous units were sampled (76 specimens) and analyzed aboard ship (see "Paleomagnetism" section, Shipboard Scientific Party, 1994). Shore-based measurements of 74 additional specimens (Table 1) confirm previous results and directly or indirectly (using thermal remagnetization, see below) extend the measured data to nine additional units.

Paleomagnetic procedures were similar to those used aboard ship and are described in Shipboard Scientific Party (1994). Natural remanent magnetization (NRM) was measured using a Molspin spinner magnetometer.

${ }^{1}$ Saunders, A.D., Larsen, H.C., and Wise, S.W., Jr. (Eds.), 1998. Proc. ODP, Sci. Results, 152: College Station, TX (Ocean Drilling Program).

${ }^{2}$ Laboratoire des Géosciences de l'Environnement, Université d'Aix-Marseille III, Arbois, CEREGE, BP 80,13545 Aix en Provence Cedex 4, France. vandamme@cerege.fr

${ }^{3}$ Department of Oceanography, The University, Southampton, SO17 1BJ, United Kingdom.
Alternating field (AF) demagnetization was applied to 43 discrete samples using a Molspin demagnetizer. The demagnetizing field was increased in 5-mT increments up to $40 \mathrm{mT}$, then in 10-mT increments up to $60 \mathrm{mT}$, and finally in $20-\mathrm{mT}$ increments up to $100 \mathrm{mT}$. The samples of the Lower Series (as defined in the "Igneous Petrology" section, Shipboard Scientific Party, 1994) were generally demagnetized in increments of $2.5 \mathrm{mT}$ up to $20 \mathrm{mT}$ to clearly discriminate any drilling-induced components.

Thirty-one samples were demagnetized by heating in a Pyrox nonmagnetic furnace. Ten samples were heated as part of a paleointensity experiment (Thellier and Thellier, 1959) to test the thermoremanent origin of the magnetization of flows 61-65. Thirteen samples were treated by heating to determine partial remagnetization by overlying flows. Eight other samples were heated to eliminate possible secondary magnetic components resulting from alteration when oxidation was clearly observable.

The demagnetization measurements were analyzed on Zijderveld (1967) plots. The magnetic components were determined using the least-squares method of Kirschvink (1980).

Magnetic susceptibility was measured aboard ship using a Bartington susceptibility meter MS2 at low frequency. Shore-based measurements were conducted using a Geofyzika Kappabridge KLY2.

\section{ATTEMPT TO REORIENT CORE PIECES}

An important problem for paleomagnetists in studies of cored hard material is the loss of half of the remanent magnetic signal: the declination. Indeed, during drilling, hard rock pieces rotate and polish themselves in the core barrel. The magnetic declination of individual pieces is lost because of their rotation during coring. The relative declination of successive pieces is also lost: their mutual polishing destroys their physical adjustment and consequently their relative orientation.

A reorientation of core pieces was attempted by comparing the fracture orientation directly measured on cores (sample coordinates) with borehole measurements (geographic coordinates) obtained using the Formation MicroScanner (FMS; see Cambray, this volume). The FMS performs high-resolution measurements of electrical resistivity, permitting the identification of fractures and providing their orientation (related to magnetic north) by means of an internal triaxial 
Table 1. Paleomagnetic results obtained on discrete samples.

\begin{tabular}{|c|c|c|c|c|c|c|c|c|}
\hline $\begin{array}{l}\text { Core, section, } \\
\text { interval }(\mathrm{cm})\end{array}$ & Piece & $\operatorname{Tr}$ & $\begin{array}{l}\text { Depth } \\
\text { (mbsf) }\end{array}$ & $\begin{array}{l}\text { NRM } \\
(\mathrm{A} / \mathrm{m})\end{array}$ & $\begin{array}{c}\mathrm{X} \\
\left(10^{-3} \mathrm{SI}\right)\end{array}$ & Q ratio & $\begin{array}{c}\text { Ch inc } \\
\left({ }^{\circ}\right)\end{array}$ & Unit \\
\hline \multicolumn{9}{|l|}{ 152-917A- } \\
\hline $\begin{array}{l}\text { Upper Series } \\
\text { 6R-1,94-96 }\end{array}$ & 9 & $\mathrm{~s}$ & \multicolumn{5}{|c|}{ Upper Series } & 1 \\
\hline $7 \mathrm{R}-1,140-143$ & 22 & $\mathrm{~s}$ & 48.10 & 48.23 & 47.30 & 23.75 & -44.7 & 2 \\
\hline $8 \mathrm{R}-4,14-16$ & 2 & $\mathrm{~s}$ & 60.19 & 1.87 & 8.80 & 4.95 & -46.0 & 6 \\
\hline $9 \mathrm{R}-1,15-19$ & 3 & $\mathrm{~s}$ & 64.95 & 3.16 & 17.60 & 4.19 & -51.1 & 7 \\
\hline $9 \mathrm{R}-3,58-63$ & $7 \mathrm{~A}$ & $\mathrm{~s}$ & 68.19 & 6.45 & 23.70 & 6.33 & -69.1 & 8 \\
\hline $10 \mathrm{R}-1,66-68$ & $1 \mathrm{~F}$ & $\mathrm{~s}$ & 74.56 & 5.68 & 21.10 & 6.27 & -76.0 & 9 \\
\hline $10 \mathrm{R}-4,52-56$ & $2 \mathrm{~B}$ & $\mathrm{~s}$ & 78.57 & 6.90 & 25.20 & 6.36 & -80.9 & 10 \\
\hline $10 \mathrm{R}-5,121-125$ & $5 \mathrm{~A}$ & $\mathrm{~s}$ & 80.76 & 19.71 & 32.00 & 14.33 & -79.0 & 11 \\
\hline $11 \mathrm{R}-4,31-35$ & 1B & $\mathrm{s}$ & 87.07 & 5.75 & 16.90 & 7.93 & -80.3 & 13 \\
\hline $12 \mathrm{R}-1,35-39$ & $4 \mathrm{~A}$ & s & 92.35 & 0.87 & 17.20 & 1.18 & -39.0 & 14 \\
\hline $13 \mathrm{R}-1,77-81$ & 4 & $\mathrm{~s}$ & 101.87 & 0.77 & 7.41 & 2.41 & -74.9 & 16 \\
\hline $13 \mathrm{R}-5,107-111$ & 10 & $\mathrm{~s}$ & 108.06 & 1.49 & 6.53 & 5.31 & -64.4 & 17 \\
\hline 13R-6, 48-50 & 5 & $\mathrm{t}$ & 108.91 & 4.31 & 14.30 & 6.99 & -64.7 & $17^{*}$ \\
\hline $14 \mathrm{R}-2,61-63$ & $1 \mathrm{C}$ & a & 111.86 & 1.43 & 2.90 & 11.48 & -58.1 & 18 \\
\hline $14 \mathrm{R}-3,97-101$ & 7 & $\mathrm{~s}$ & 113.62 & 1.29 & 6.60 & 4.54 & -50.7 & 18 \\
\hline $14 \mathrm{R}-4,83-88$ & $2 \mathrm{~A}$ & $\mathrm{~s}$ & 114.52 & 1.50 & 7.31 & 4.78 & -52.1 & 18 \\
\hline $15 \mathrm{R}-2,5-9$ & $1 \mathrm{~A}$ & $\mathrm{~s}$ & 120.70 & 2.38 & 10.50 & 5.26 & -68.8 & 19 \\
\hline $15 \mathrm{R}-2,44-46$ & $2 \mathrm{~A}$ & a & 121.09 & 4.24 & 6.69 & 14.75 & -68.5 & 19 \\
\hline $16 \mathrm{R}-1,70-72$ & $3 B$ & $\mathrm{t}$ & 129.00 & 1.47 & 1.77 & 19.41 & $\begin{array}{l}-56.6 \\
-66.0\end{array}$ & $20^{*}$ \\
\hline $16 \mathrm{R}-2,108-110$ & $3 \mathrm{C}$ & a & 130.79 & 1.60 & 6.19 & 6.00 & -70.0 & 21 \\
\hline $16 \mathrm{R}-3,74-76$ & $3 \mathrm{~B}$ & a & 131.85 & 0.60 & 2.82 & 4.93 & -52.5 & 21 \\
\hline $16 \mathrm{R}-4,59-63$ & $8 \mathrm{~A}$ & $\mathrm{~s}$ & 133.13 & 0.52 & 3.38 & 3.60 & -46.6 & 21 \\
\hline 16R-6, 42-74 & 5 & $\mathrm{t}$ & 136.23 & 26.71 & 19.90 & 31.16 & -51.1 & $21^{*}$ \\
\hline $17 \mathrm{R}-2,50-52$ & 1 & a & 138.67 & 5.74 & 14.10 & 9.51 & -44.9 & 23 \\
\hline 17R-2, 72-76 & 2 & $\mathrm{~s}$ & 138.89 & 6.65 & 33.20 & 4.67 & -43.8 & 23 \\
\hline $17 \mathrm{R}-4,35-37$ & $3 \mathrm{~A}$ & a & 141.04 & 4.51 & 9.37 & 11.20 & -40.6 & 24 \\
\hline 17R-4, 73-77 & $3 \mathrm{~B}$ & s & 141.42 & 5.09 & 23.60 & 5.03 & -38.8 & 24 \\
\hline $18 \mathrm{R}-2,40-42$ & 2 & $\mathrm{t}$ & 147.85 & 3.06 & 5.75 & 12.40 & -61.5 & 25 \\
\hline $18 \mathrm{R}-3,32-36$ & 1 & s & 148.90 & 2.93 & 9.30 & 7.34 & -60.8 & 25 \\
\hline $18 \mathrm{R}-3,127-129$ & 2 & $\mathrm{a}$ & 149.85 & 2.67 & 3.68 & 16.86 & 63.0 & 25 \\
\hline $18 \mathrm{R}-4,95-99$ & $7 \mathrm{~A}$ & $\mathrm{~s}$ & 150.94 & 1.76 & 17.00 & 2.41 & -60.0 & 26 \\
\hline $18 \mathrm{R}-4,116-118$ & $7 \mathrm{C}$ & $\mathrm{t}$ & 151.15 & 2.56 & 10.30 & 5.80 & -59.9 & 26 \\
\hline $18 \mathrm{R}-6,36-38$ & 4 & $\mathrm{a}$ & 153.30 & 2.04 & 5.06 & 9.39 & -64.0 & 27 \\
\hline $18 \mathrm{R}-6,97-101$ & $5 \mathrm{~A}$ & $\mathrm{~s}$ & 153.91 & 2.56 & 8.86 & 6.72 & -61.5 & 27 \\
\hline 19R-1, 69-71 & $3 \mathrm{~A}$ & $\mathrm{t}$ & 155.99 & 6.63 & 10.90 & 14.12 & -62.3 & $28-29$ \\
\hline 19R-2, 41-46 & 1B & $\mathrm{s}$ & 157.11 & 3.13 & 8.95 & 8.13 & -63.6 & 30 \\
\hline $19 \mathrm{R}-4,44-48$ & $2 \mathrm{~B}$ & $\mathrm{~s}$ & 160.03 & 1.62 & 6.11 & 6.16 & -61.3 & 31 \\
\hline $20 \mathrm{R}-1,21-26$ & $2 \mathrm{~B}$ & $\mathrm{~s}$ & 164.71 & 2.09 & 6.35 & 7.67 & -62.3 & 31 \\
\hline $20 \mathrm{R}-3,6-8$ & $1 \mathrm{~A}$ & a & 167.56 & 0.65 & 2.01 & 7.59 & -46.9 & 32 \\
\hline 20R-5, 36-41 & 4 & s & 170.50 & 3.60 & 7.73 & 10.86 & -46.8 & 32 \\
\hline $21 \mathrm{R}-3,138-142$ & 5 & $\mathrm{~s}$ & 177.87 & 1.89 & 33.40 & 1.32 & -21.7 & 33 \\
\hline $21 \mathrm{R}-5,133-135$ & 7 & $\mathrm{t}$ & 180.72 & 3.26 & 35.90 & 2.12 & -36.4 & 33 \\
\hline $21 \mathrm{R}-6,15-17$ & 1 & a & 180.92 & 1.73 & 4.85 & 8.29 & -40.5 & 33 \\
\hline Middle Series & & & & & & & & \\
\hline $23 \mathrm{R}-1,53-55$ & 6 & $\mathrm{t}$ & 192.43 & 1.46 & 36.40 & 0.93 & -12.6 & 34 \\
\hline $23 \mathrm{R}-1,68-73$ & 9 & s & 192.58 & 2.56 & 54.00 & 1.10 & $(-2.8)$ & $34 \mathrm{~B}$ \\
\hline $25 \mathrm{R}-1,82-86$ & $9 \mathrm{~A}$ & s & 207.12 & 1.17 & 26.90 & 1.01 & -65.0 & 36 \\
\hline $25 \mathrm{R}-1,137-139$ & $11 \mathrm{C}$ & $\mathrm{a}$ & 207.67 & 0.99 & 17.10 & 1.35 & -64.0 & 36 \\
\hline 25R-2, 72-74 & $8 \mathrm{~A}$ & $\mathrm{t}$ & 208.49 & 1.57 & 19.90 & 1.84 & -64.4 & $36^{*}$ \\
\hline $26 \mathrm{R}-1,113-115$ & 15 & a & 211.93 & 3.32 & 8.47 & 9.12 & -62.8 & 38 \\
\hline $26 \mathrm{R}-2,5-11$ & 1 & $\mathrm{~s}$ & 212.32 & 2.69 & 17.00 & 3.68 & -65.9 & 38 \\
\hline $27 \mathrm{R}-1,70-75$ & $5 \mathrm{~B}$ & $\mathrm{~s}$ & 216.00 & 4.38 & 18.40 & 5.53 & -62.3 & 40 \\
\hline $27 \mathrm{R}-1,125-127$ & 11 & $\mathrm{a}$ & 216.55 & 3.23 & 12.30 & 6.13 & -65.2 & 40 \\
\hline $27 \mathrm{R}-2,85-88$ & $9 \mathrm{~A}$ & $\mathrm{a}$ & 217.62 & 3.28 & 18.50 & 4.12 & -72.3 & 41 \\
\hline $28 \mathrm{R}-1,87-89$ & $8 \mathrm{~A}$ & $\mathrm{a}$ & 221.17 & 1.77 & 9.19 & 4.48 & -79.6 & 42 \\
\hline $29 \mathrm{R}-3,13-15$ & 2 & $\mathrm{a}$ & 228.03 & 3.79 & 18.50 & 4.76 & -59.0 & 43 \\
\hline $30 \mathrm{R}-1,110-115$ & 15 & $\mathrm{~s}$ & 231.00 & 1.49 & 17.70 & 1.95 & -62.1 & 43 \\
\hline $30 \mathrm{R}-2,82-84$ & 4 & $\mathrm{t}$ & 231.99 & 2.38 & 19.10 & 2.90 & -60.2 & $43^{*}$ \\
\hline $30 \mathrm{R}-3,37-41$ & 6 & $\mathrm{~s}$ & 233.02 & 4.65 & 23.70 & 4.56 & -63.1 & 44 \\
\hline $31 \mathrm{R}-2,73-75$ & 11 & $\mathrm{t}$ & 236.83 & 3.14 & 15.80 & 4.64 & -48.4 & $45^{*}$ \\
\hline $31 \mathrm{R}-3,106-110$ & $11 \mathrm{~A}$ & $\mathrm{~s}$ & 238.66 & 3.26 & 9.18 & 8.26 & -60.8 & 46 \\
\hline $32 \mathrm{R}-1,30-32$ & $2 \mathrm{~B}$ & a & 239.90 & 2.44 & 4.14 & 13.72 & -54.9 & 46 \\
\hline $32 \mathrm{R}-2,130-132$ & $20 \mathrm{~A}$ & $\mathrm{t}$ & 242.40 & 10.02 & 9.13 & 25.54 & $(-16.6)$ & $?$ \\
\hline $32 \mathrm{R}-3,134-137$ & 13 & $\mathrm{~s}$ & 243.90 & 5.36 & 19.20 & 6.50 & -55.3 & 47 \\
\hline $33 \mathrm{R}-2,40-42$ & 6 & a & 251.10 & 9.73 & 15.80 & 14.31 & -45.4 & 48 \\
\hline $34 \mathrm{R}-1,19-21$ & 4 & $\mathrm{~s}$ & 259.09 & 2.29 & 24.50 & 2.17 & -61.9 & 49 \\
\hline $34 \mathrm{R}-2,41-46$ & $4 \mathrm{~A}$ & $\mathrm{~s}$ & 260.75 & 3.09 & 54.40 & 1.32 & $(+53.3)$ & 49 \\
\hline $35 \mathrm{R}-2,5-7$ & $1 \mathrm{~A}$ & $\mathrm{a}$ & 264.85 & 2.83 & 8.10 & 8.13 & -79.0 & 50 \\
\hline $35 \mathrm{R}-2,26-31$ & $1 \mathrm{~B}$ & $\mathrm{~s}$ & 265.06 & 2.55 & 14.00 & 4.25 & -75.5 & 50 \\
\hline $36 \mathrm{R}-6,34-40$ & 1B & $\mathrm{s}$ & 275.56 & 13.39 & 27.20 & 11.44 & -69.1 & 52 \\
\hline $37 \mathrm{R}-3,22-24$ & 3 & $\mathrm{a}$ & 281.16 & 4.83 & 15.60 & 7.21 & -80.7 & 52 \\
\hline $38 \mathrm{R}-2,64-66$ & 1B & $\mathrm{a}$ & 289.69 & 0.45 & 35.50 & 0.30 & -81.0 & 52 \\
\hline $39 \mathrm{R}-3,95-100$ & $6 \mathrm{C}$ & $\mathrm{s}$ & 296.14 & 3.22 & 18.30 & 4.10 & -76.8 & 52 \\
\hline $41 \mathrm{R}-2,75-77$ & $3 \mathrm{~B}$ & $\mathrm{p}$ & 309.20 & 2.81 & 18.50 & 3.53 & -84.6 & 52 \\
\hline $47 \mathrm{R}-1,46-48$ & $1 \mathrm{D}$ & $\mathrm{t}$ & 346.16 & 7.38 & 5.69 & 30.19 & -85.9 & $53 *$ \\
\hline $47 \mathrm{R}-3,80-82$ & $5 \mathrm{D}$ & $\mathrm{s}$ & 34943 & 10.46 & 13.70 & 17.80 & $\begin{array}{l}-76.4 \\
-78.9\end{array}$ & $\begin{array}{l}54 \\
54\end{array}$ \\
\hline Lower Series & & & & & & & & \\
\hline $\begin{array}{l}\text { Lower serres } \\
54 \mathrm{R}-2,61-63\end{array}$ & $1 \mathrm{~A}$ & a & 38623 & 181 & 203 & 1430 & 673 & 58 \\
\hline $54 \mathrm{R}-4,88-90$ & $1 \mathrm{C}$ & $\mathrm{p}$ & $\begin{array}{l}380.23 \\
389.13\end{array}$ & $\begin{array}{l}1.01 \\
0.29\end{array}$ & 5.16 & $\begin{array}{r}14.39 \\
1.29\end{array}$ & $\begin{array}{l}-0 / .3 \\
-73.2\end{array}$ & $\begin{array}{l}58 \\
58\end{array}$ \\
\hline $54 \mathrm{R}-5,16-18$ & $1 \mathrm{~A}$ & $\mathrm{~s}$ & 389.84 & 0.32 & 11.20 & 0.66 & -74.8 & $\begin{array}{l}30 \\
58\end{array}$ \\
\hline $55 \mathrm{R}-2,82-84$ & $1 \mathrm{~F}$ & $\mathrm{p}$ & 396.11 & 0.27 & 15.60 & 0.40 & 75.1 & 60 \\
\hline $55 \mathrm{R}-3,101-103$ & 1I & a & 397.45 & 1.76 & 31.90 & 1.28 & -73.1 & 60 \\
\hline $55 \mathrm{R}-4,11-14$ & 1B & $\mathrm{s}$ & 398.05 & 5.98 & 52.70 & 2.64 & 56.7 & 60 \\
\hline
\end{tabular}


Table 1 (continued).

\begin{tabular}{|c|c|c|c|c|c|c|c|c|}
\hline $\begin{array}{l}\text { Core, section, } \\
\text { interval }(\mathrm{cm})\end{array}$ & Piece & $\operatorname{Tr}$ & $\begin{array}{l}\text { Depth } \\
\text { (mbsf) }\end{array}$ & $\begin{array}{l}\mathrm{NRM} \\
(\mathrm{A} / \mathrm{m})\end{array}$ & $\begin{array}{c}\mathrm{X} \\
\left(10^{-3} \mathrm{SI}\right)\end{array}$ & Q ratio & $\begin{array}{c}\mathrm{Ch} \text { inc } \\
\left({ }^{\circ}\right)\end{array}$ & Unit \\
\hline $57 \mathrm{R}-1,111-113$ & 6 & $\mathrm{a}$ & 414.31 & 0.10 & 0.87 & 2.79 & 13.2 & 61 \\
\hline $57 \mathrm{R}-2,91-93$ & $3 \mathrm{C}$ & $\mathrm{p}$ & 415.59 & 0.16 & 0.80 & 4.82 & 6.7 & 1 \\
\hline $57 \mathrm{R}-3,81-83$ & 1 & $\mathrm{~s}$ & 416.30 & 0.13 & 2.45 & 1.21 & $(-23.3)$ & $61 \mathrm{~B}$ \\
\hline $57 \mathrm{R}-6,63-65$ & $2 \mathrm{~B}$ & $\mathrm{p}$ & 420.44 & 1.58 & 7.95 & 4.64 & 10.9 & 62 \\
\hline 57R-6, 89-91 & $2 \mathrm{C}$ & $\mathrm{s}$ & 420.70 & 0.99 & 13.30 & 1.72 & 8.2 & 62 \\
\hline $57 \mathrm{R}-7,12-14$ & $1 \mathrm{~A}$ & $\mathrm{a}$ & 421.41 & 0.29 & 1.98 & 3.36 & 6.2 & 62 \\
\hline $57 \mathrm{R}-7,20-22$ & $1 \mathrm{~A}$ & $\mathrm{~s}$ & 421.49 & 1.05 & 4.89 & 4.99 & 11.3 & 62 \\
\hline $58 \mathrm{R}-2,33-37$ & $1 \mathrm{~B}$ & $\mathrm{~s}$ & 423.89 & 0.36 & 11.70 & 0.71 & 4.1 & 63 \\
\hline $58 \mathrm{R}-2,61-63$ & $2 \mathrm{~A}$ & $\mathrm{a}$ & 424.17 & 0.41 & 4.90 & 1.97 & 8.8 & 63 \\
\hline $58 \mathrm{R}-3,21-23$ & $1 \mathrm{~A}$ & $\mathrm{p}$ & 424.90 & 2.07 & 9.12 & 5.28 & 11.5 & 63 \\
\hline 58R-4, 39-42 & $2 \mathrm{~A}$ & $\mathrm{t}$ & 426.49 & 0.44 & 0.70 & 14.72 & -74.2 & 64 \\
\hline $59 \mathrm{R}-1,64-66$ & $2 \mathrm{~F}$ & $\mathrm{p}$ & 432.74 & 0.52 & 5.19 & 2.31 & -65.5 & 64 \\
\hline $59 \mathrm{R}-1,102-109$ & $2 \mathrm{~F}$ & s & 433.12 & 0.43 & 6.36 & 1.57 & -70.6 & 64 \\
\hline $60 \mathrm{R}-1,75-77$ & $2 \mathrm{~F}$ & $\mathrm{~s}$ & 437.45 & 3.49 & 25.30 & 3.20 & -29.2 & 65 \\
\hline $61 \mathrm{R}-2,54-56$ & 1 & $\mathrm{~s}$ & 443.16 & 0.03 & 3.70 & 0.16 & -74.7 & 66 \\
\hline $61 \mathrm{R}-3,54-56$ & $2 \mathrm{~B}$ & a & 444.66 & 0.05 & 2.24 & 0.55 & -76.8 & 66 \\
\hline $62 \mathrm{R}-1,64-66$ & $5 \mathrm{~B}$ & $\mathrm{p}$ & 451.94 & 0.31 & 3.47 & 2.07 & -64.3 & 66 \\
\hline $64 \mathrm{R}-1,125-127$ & 5 & $\mathrm{~s}$ & 471.95 & 4.04 & 17.00 & 5.55 & -73.0 & 68 \\
\hline $64 \mathrm{R}-2,85-87$ & $3 \mathrm{D}$ & $\mathrm{a}$ & 473.05 & 1.66 & 5.83 & 6.62 & -73.0 & 68 \\
\hline $65 \mathrm{R}-3,7-9$ & $1 \mathrm{~A}$ & $\mathrm{a}$ & 483.20 & 2.07 & 5.61 & 8.57 & -81.6 & 69 \\
\hline $67 R-2,102-104$ & $9 \mathrm{C}$ & $\mathrm{a}$ & 502.06 & 1.78 & 7.32 & 5.64 & -79.3 & 70 \\
\hline $67 \mathrm{R}-3,100-102$ & $6 \mathrm{~A}$ & $\mathrm{p}$ & 503.43 & 2.08 & 7.97 & 6.06 & -79.0 & 70 \\
\hline $67 \mathrm{R}-4,106-110$ & $5 \mathrm{~A}$ & $\mathrm{~s}$ & 504.99 & 2.53 & 16.10 & 3.66 & -79.5 & 70 \\
\hline $69 R-2,90-94$ & $3 \mathrm{D}$ & $\mathrm{s}$ & 521.20 & 5.90 & 11.90 & 11.53 & -70.1 & 71 \\
\hline $69 \mathrm{R}-3,25-27$ & $1 \mathrm{~B}$ & $\mathrm{a}$ & 521.83 & 2.97 & 3.73 & 18.54 & -69.0 & 71 \\
\hline $71 \mathrm{R}-4,32-34$ & 3 & $\mathrm{~s}$ & 542.12 & 2.94 & 10.70 & 6.39 & -64.8 & 72 \\
\hline $72 \mathrm{R}-1,100-102$ & $3 \mathrm{E}$ & $\mathrm{s}$ & 548.30 & 2.04 & 11.10 & 4.27 & -66.5 & 73 \\
\hline $72 \mathrm{R}-3,14-16$ & 1 & $\mathrm{t}$ & 550.22 & 2.85 & 8.84 & 7.50 & -60.7 & 73 \\
\hline $72 R-4,31-33$ & $1 \mathrm{~B}$ & a & 551.81 & 0.52 & 8.43 & 1.45 & -66.4 & 73 \\
\hline $73 \mathrm{R}-1,23-25$ & $3 \mathrm{~A}$ & $\mathrm{t}$ & 557.23 & 6.49 & 36.60 & 4.13 & $(-42.9)$ & $?$ \\
\hline $73 \mathrm{R}-2,42-44$ & $1 \mathrm{D}$ & $\mathrm{s}$ & 558.83 & 7.68 & 74.20 & 2.41 & -78.2 & 74 \\
\hline $78 \mathrm{R}-2,136-137$ & $4 \mathrm{~B}$ & $\mathrm{~s}$ & 597.99 & 0.93 & 3.65 & 5.96 & -68.5 & 76 \\
\hline $78 \mathrm{R}-3,65-67$ & $1 \mathrm{D}$ & $\mathrm{a}$ & 598.70 & 1.09 & 3.38 & 7.48 & -66.7 & 76 \\
\hline 78R-7, $18-20$ & $1 \mathrm{~A}$ & $\mathrm{t}$ & 603.57 & 18.30 & 8.73 & 48.80 & -67.5 & $76^{*}$ \\
\hline $79 \mathrm{R}-2,91-95$ & $1 \mathrm{E}$ & $\mathrm{s}$ & 607.35 & 1.24 & 11.10 & 2.60 & -62.3 & 78 \\
\hline $79 \mathrm{R}-3,28-30$ & $1 \mathrm{C}$ & a & 608.15 & 4.62 & 5.65 & 19.02 & -56.3 & 78 \\
\hline 79R-5, 90-92 & $1 \mathrm{E}$ & $\mathrm{s}$ & 611.37 & 0.17 & 14.00 & 0.28 & -63.9 & 79 \\
\hline $80 \mathrm{R}-3,57-59$ & $1 \mathrm{D}$ & $\mathrm{a}$ & 618.17 & 2.55 & 3.71 & 15.97 & -60.1 & 79 \\
\hline $80 \mathrm{R}-4,102-106$ & $1 \mathrm{D}$ & $\mathrm{s}$ & 619.91 & 1.97 & 7.46 & 6.13 & -58.7 & 79 \\
\hline $80 \mathrm{R}-6,9-11$ & $1 \mathrm{~A}$ & $\mathrm{p}$ & 621.86 & 1.82 & 5.56 & 7.62 & -56.4 & 79 \\
\hline $81 \mathrm{R}-3,61-63$ & $1 \mathrm{C}$ & a & 627.89 & 0.62 & 4.01 & 3.60 & $(8.8)$ & 80 \\
\hline 81R-3, 97-99 & $1 \mathrm{D}$ & $\mathrm{s}$ & 628.25 & 0.31 & 2.41 & 2.99 & -68.1 & 80 \\
\hline $82 \mathrm{R}-2,109-111$ & $1 \mathrm{C}$ & $\mathrm{s}$ & 636.66 & 0.91 & 5.84 & 3.63 & -70.8 & 82 \\
\hline $83 \mathrm{R}-2,129-131$ & $1 \mathrm{D}$ & $\mathrm{s}$ & 646.59 & 0.95 & 8.24 & 2.67 & -65.7 & 82 \\
\hline $83 R-3,125-127$ & $7 \mathrm{C}$ & $\mathrm{a}$ & 648.00 & 0.18 & 0.69 & 6.10 & -67.6 & 82 \\
\hline $84 \mathrm{R}-2,95-97$ & $3 \mathrm{D}$ & $\mathrm{s}$ & 655.95 & 2.40 & 18.30 & 3.05 & -66.3 & 83 \\
\hline $85 \mathrm{R}-2,109-111$ & 10 & $\mathrm{t}$ & 665.72 & 1.07 & 6.58 & 3.80 & -78.7 & 84 \\
\hline $86 \mathrm{R}-1,21-23$ & 2 & a & 673.11 & 0.77 & 2.95 & 6.11 & 75.5 & 84 \\
\hline $86 \mathrm{R}-3,66-70$ & $1 \mathrm{D}$ & $\mathrm{s}$ & 675.99 & 0.86 & 4.96 & 4.03 & -70.6 & 84 \\
\hline $87 \mathrm{R}-2,31-33$ & $1 \mathrm{~A}$ & $\mathrm{~s}$ & 683.59 & 5.98 & 13.20 & 10.58 & -73.4 & 84 \\
\hline $89 \mathrm{R}-1,39-41$ & $1 \mathrm{C}$ & $\mathrm{a}$ & 701.79 & 0.30 & 3.15 & 2.18 & -69.2 & 84 \\
\hline $89 \mathrm{R}-2,6-8$ & $1 \mathrm{~B}$ & $\mathrm{~s}$ & 702.35 & 0.43 & 6.43 & 1.57 & -73.6 & 84 \\
\hline $90 \mathrm{R}-1,26-28$ & $2 \mathrm{~A}$ & $\mathrm{t}$ & 710.36 & 1.17 & 1.49 & 18.31 & -78.0 & 85 \\
\hline $90 \mathrm{R}-1,41-45$ & $2 \mathrm{~A}$ & $\mathrm{~s}$ & 710.51 & 1.22 & 3.01 & 9.43 & -80.1 & 85 \\
\hline 90R-4, 91-93 & 1 & a & 714.67 & 1.55 & 2.02 & 17.83 & -76.8 & 86 \\
\hline $90 \mathrm{R}-5,52-56$ & $1 \mathrm{~B}$ & $\mathrm{~s}$ & 715.60 & 1.12 & 5.44 & 4.81 & -77.2 & 86 \\
\hline $92 \mathrm{R}-4,5-10$ & 1 & $\mathrm{~s}$ & 727.44 & 0.78 & 7.00 & 2.60 & -55.7 & 87 \\
\hline 93R-2, 88-90 & $1 \mathrm{D}$ & $\mathrm{a}$ & 732.62 & 1.03 & 4.08 & 5.87 & -49.0 & 87 \\
\hline $94 \mathrm{R}-4,8-10$ & $1 \mathrm{~A}$ & $\mathrm{~s}$ & 744.11 & 0.98 & 8.78 & 2.60 & -49.6 & 87 \\
\hline $95 \mathrm{R}-2,61-63$ & $1 \mathrm{D}$ & $\mathrm{s}$ & 751.91 & 0.89 & 6.56 & 3.17 & -47.3 & 87 \\
\hline $96 \mathrm{R}-1,123-125$ & $10 \mathrm{~A}$ & $\mathrm{~s}$ & 760.63 & 0.84 & 12.20 & 1.60 & -76.4 & 88 \\
\hline $97 \mathrm{R}-2,42-44$ & 1B & $\mathrm{s}$ & 770.67 & 9.38 & 5.70 & 38.31 & -65.9 & 89 \\
\hline $97 \mathrm{R}-3,6-8$ & 1 & $\mathrm{a}$ & 771.61 & 1.15 & 2.86 & 9.34 & -69.8 & 89 \\
\hline $99 \mathrm{R}-2,23-26$ & $1 \mathrm{~B}$ & a & 790.00 & 1.22 & 11.50 & 2.46 & -76.9 & 90 \\
\hline $99 \mathrm{R}-2,78-80$ & $1 \mathrm{~F}$ & $\mathrm{~s}$ & 790.55 & 3.26 & 14.70 & 5.15 & -78.3 & 90 \\
\hline 100R-1, 104-108 & $1 \mathrm{~F}$ & $\mathrm{~s}$ & 799.04 & 1.02 & 13.10 & 1.82 & -73.9 & 91 \\
\hline $100 \mathrm{R}-3,80-82$ & $1 \mathrm{C}$ & $\mathrm{a}$ & 801.37 & 2.45 & 6.06 & 9.41 & -75.8 & 91 \\
\hline $101 \mathrm{R}-4,81-85$ & 5 & $\mathrm{~s}$ & 812.24 & 3.02 & 18.30 & 3.83 & -78.1 & 92 \\
\hline $102 \mathrm{R}-2,38-40$ & $1 \mathrm{~B}$ & $\mathrm{a}$ & 819.05 & 5.90 & 8.42 & 16.31 & -71.6 & 92 \\
\hline
\end{tabular}

Notes: The table is divided into three parts corresponding to petrologic series ("Igneous Petrology," Shipboard Scientific Party, 1994). Tr = treatment applied on samples; s: shipboard measurements (alternating field treatment); a: alternating field treatment; t: thermal treatment; p: paleointensity experiment (direction determined after 20-mT alternating field treatment, see text). NRM = intensity of natural remanent magnetization. $\mathrm{X}=$ volumetric magnetic susceptibility. $\mathrm{Q}$ ratio $=$ Koenigsberger ratio. Ch inc $=$ characteristic inclination; values between parentheses are not used in calculations of unit means (Table 3). Unit = identified igneous unit ("Igneous Petrology," Shipboard Scientific Party, 1994) associated to magnetic component. $*$ associated magnetic component is a thermal remagnetization by overlying flow. ? = the origin of magnetization has not been identified.

magnetometer. The correlation of fractures measured in cores with those measured in borehole by the FMS was difficult. The consistency of declination of magnetic components defined in different pieces from the same flow allows us to test the quality of fracture correlations. The results of this test were quite variable: declinations deduced from these reorientations differed from a few degrees to about $180^{\circ}$. The high density of fractures in Hole 917A material did not allow confident correlations. Consequently, magnetic declination was lost, as is usually the case with this type of material.

\section{PALEOMAGNETIC METHODS AND RESULTS}

\section{AF Demagnetization}

AF demagnetization allows the determination of a single magnetic component, which was assumed to represent the original magnetization of Cores 152-917A-6R through 43R (Fig. 1A). Below Core 152-917A-43R, the coring bit was changed twice (at Core 152-917A$44 \mathrm{R}$ and $79 \mathrm{R}$ ), and the two new drill bits induced a secondary mag- 
A

Figure 1. Examples of Zijderveld plots of AFdemagnetized samples. A. Sample with a single magnetic component. (152-917A-17R-2, 50-52 $\mathrm{cm})$ B. Sample with two magnetic components. (152-917A-89R-1, 39-41 cm) The secondary magnetization, oriented vertically downward, was induced by drilling.

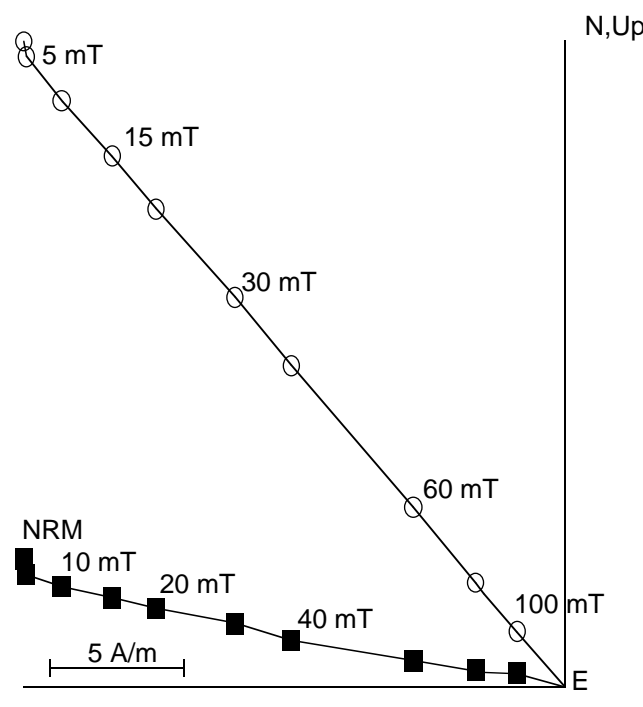

B

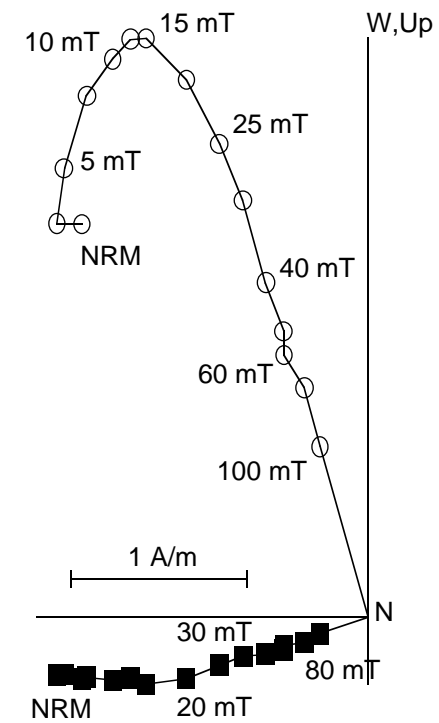

netization, oriented vertically downward, to all the cores from Core 152-917A-44R to 92R (Fig. 1B). This remagnetization, however, could be removed by an alternating field of $20 \mathrm{mT}$; consequently, the primary magnetization was easily isolated with treatments between 20 and $100 \mathrm{mT}$.

\section{Thermal Demagnetization}

We selected 14 samples located close to the top of the flow (approximately $10 \mathrm{~cm}$ of recovered core) for thermal demagnetization to determine their primary magnetization and a possible secondary magnetization resulting from heating in situ by the overlying flow. The samples from Core 152-917A-44R and below were previously AF demagnetized to $20 \mathrm{mT}$ to eliminate the drilling-induced component described above. The results of thermal demagnetization are summarized in Table 2.

Only two samples showed two components during demagnetization (Fig. 2A): Samples 152-917A-16R-1, 70-72 cm, and 152-917A47R-1, 46-48 cm, provide inclinations for flows 20 and 21, and 53 and 54, respectively. In both cases, the overlying flow inclinations are known only by these determinations. We could not reasonably sample these overlying flows because they are brecciated and altered.

The 12 other samples present a single component (Fig. 2B). We propose three different interpretations for the origin of magnetization, depending for each sample on the magnetic components of the sampled flow and the overlying flow.

First, when the magnetic components of the sampled flow and the overlying flow have already been determined directly by AF demagnetization of other samples, we compare both AF components with the result obtained from thermal demagnetization. Three scenarios are possible: (1) the inclination of the studied sample is close to that of the sampled flow and we consider the magnetization to be primary (Samples 152-917A-58R-42, 39-42 cm, 85R-2, 109-111 cm, 90R-1, $26-28 \mathrm{~cm}),(2)$ the inclination of the studied sample is close to that of the overlying flow and we conclude that a complete thermal remagnetization has occurred (Samples 152-917A-13R-6, 48-50 cm, and $30 \mathrm{R}-2,82-84 \mathrm{~cm}$ ), and (3) the inclination of the studied sample is completely different from the inclinations of the sampled and overlying flows and we ignore the result, assuming that a sampling or measuring problem has occurred (Samples 152-917A-32R-2, 130-132 $\mathrm{cm}$, and $73 \mathrm{R}-1,23-25 \mathrm{~cm}$ ).
Table 2. Summary of thermal demagnetization behaviors.

\begin{tabular}{lcccccc}
\hline $\begin{array}{c}\text { Core, section, } \\
\text { interval }(\mathrm{cm})\end{array}$ & Piece & $\begin{array}{c}\text { Sampled } \\
\text { unit }\end{array}$ & $\begin{array}{c}\text { Measured } \\
\text { unit }\end{array}$ & $\begin{array}{c}\text { Known } \\
\text { unit }\end{array}$ & $\begin{array}{c}\text { Determined } \\
\text { component }\end{array}$ & $\begin{array}{c}\text { New } \\
\text { unit }\end{array}$ \\
\hline 152-917A- & & & & & & \\
13R-6, 48-50 & 5 & 18 & 17 & Both & 2 & \\
16R-1, 70-72 & 3B & 21 & $20+21$ & 21 & $1+2$ & 20 \\
16R-6, 42-74 & 5 & 22 & 21 & 21 & 2 & \\
19R-1, 69-71 & 3A & 29 & 28 & None & 2 & 28 \\
25R-2, 72-74 & $8 \mathrm{~A}$ & 37 & 36 & 36 & 2 & \\
30R-2, 82-84 & 4 & 44 & 43 & Both & 2 & \\
31R-2, 73-75 & 11 & 46 & 45 & 46 & 2 & 45 \\
32R-2, 130-132 & 20A & 47 & $?$ & Both & X & \\
47R-1, 46-48 & 1D & 54 & $53+54$ & 54 & $1+2$ & 53 \\
58R-4, 39-42 & 2A & 64 & 64 & Both & 1 & \\
73R-1, 23-25 & 3A & 74 & $?$ & Both & X & \\
78R-7, 18-20 & 1A & 77 & 76 & 76 & 2 & \\
85R-2, 109-111 & 10 & 84 & 84 & Both & 1 & \\
90R-1, 26-28 & 2A & 85 & 85 & Both & 1 & \\
\end{tabular}

Notes: Measured unit: igneous unit associated with the determined magnetic component. Known unit: igneous unit for which inclination is already known by direct measurements on one or several other samples; both means that magnetization of sampled and overlying units is already known; none means that neither sampled unit magnetization nor overlying unit one are known. Determined component: $1=$ primary magnetization; 2 = secondary magnetization (thermal remagnetization); $\mathrm{X}=$ no identified magnetization.

Second, when only one of the two magnetic components (that of the sampled flow or that of the overlying flow) is known thanks to AF demagnetization, we compare it with the magnetic component obtained by thermal demagnetization. There are two possibilities: (1) Samples 152-917A-16R-6, 72-74 cm, 25R-2, 72-74 cm, and 78R-7, 18-20 cm, are considered remagnetized because of the similarity between their characteristic inclinations and that of the overlying flow; and (2) Sample 152-917A-31R-2, 73-75 cm, is also considered remagnetized, because of the difference between its characteristic inclination and that of the sampled flow.

Finally, Sample 152-917A-19R-1, 69-71 cm, shows only one magnetic component. It can be the primary magnetization of Unit 29 or a complete thermal remagnetization due to reheating by the overlying Unit 28. As we have not any other sample in both units, it is impossible to compare the magnetization of Sample 152-917A-19R-1, 69-71 cm, either to the overlying flow (Unit 28) magnetization or to that of the sampled flow (Unit 29). We have so indicated in Table 1 
A

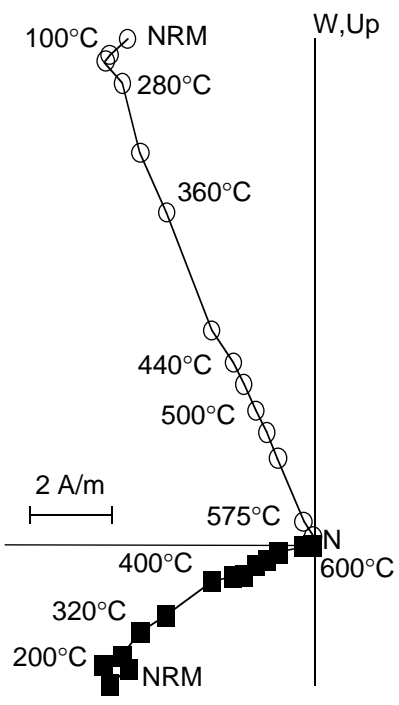

B

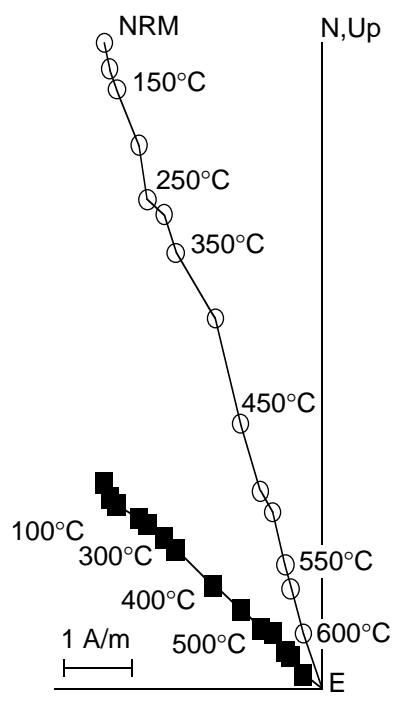

Figure 2. Examples of Zijderveld plots of thermally demagnetized samples. A. Sample showing a single magnetic component. (152-917A-30R-2, 82-84 $\mathrm{cm})$ B. Sample showing two thermal magnetic components. (152-917A-16R$1,70-72 \mathrm{~cm})$

both igneous units as possible origin for magnetization of this sample. However, considering the general behavior of the other samples taken at the uppermost part of flows, as is the case for Sample 152917A-19R-1, 69-71 cm, it is highly probable that this sample is completely remagnetized by the overlying flow (Unit 28).

The thermal treatment allows the determination of the magnetization of four additional flows (Units 20, 28, 45, and 53) using reheating by overlying flows. Indeed, at about $10 \mathrm{~cm}$ below the contact of two flows, a complete thermal remagnetization seems to have occurred. Only Samples 152-917A-85R-2, 109-111 cm (Unit 84), and 90R-1, 26-28 cm (Unit 85), give primary magnetizations in similar locations. One explanation for this particular behavior is the easy dispersal of heat because of the presence of shallow water during emplacement of Units 83 and 84, similar to what has been suggested for Units 52, 53, and 92 ("Igneous Petrology" section, Shipboard Scientific Party, 1994).

\section{Paleointensity Experiments}

Shipboard measurements showed shallow positive inclinations for Units 61-63 and a shallow negative inclination for Unit 65 ("Paleomagnetism" section, Shipboard Scientific Party, 1994), which can be interpreted as the record of a short geomagnetic event. We tried to use the method of paleointensity determination of Thellier and Thellier (1959) to test the thermoremanent origin of the magnetization of Units 61-63 and 65 but all the samples show a geochemical change during the experiment. It was consequently impossible to conclude on their magnetization origin.

\section{Mean Paleomagnetic Results per Igneous Unit}

The paleomagnetic results obtained from samples using AF and thermal treatments are listed in Table 1. For each unit, we accepted only an inclination range less than $10^{\circ}$. When this range was larger, the farthest inclination was eliminated, and is presented in parentheses in Table 1. Because the number of data per unit was small, we

Table 3. Paleomagnetic results per petrologic units.

\begin{tabular}{|c|c|c|c|c|c|c|c|c|}
\hline \multicolumn{3}{|c|}{ Upper series } & \multicolumn{3}{|c|}{ Middle series } & \multicolumn{3}{|c|}{ Lower series } \\
\hline Unit & $\mathrm{N}$ & $\begin{array}{c}\text { Ch inc } \\
\left({ }^{\circ}\right)\end{array}$ & Unit & $\mathrm{N}$ & $\begin{array}{c}\mathrm{Ch} \text { inc } \\
\left({ }^{\circ}\right)\end{array}$ & Unit & $\mathrm{N}$ & $\begin{array}{c}\text { Ch inc } \\
\left({ }^{\circ}\right)\end{array}$ \\
\hline 1 & 1 & -44.2 & 34 & 2 & -7.7 & 58 & 3 & -71.8 \\
\hline 2 & 1 & -44.7 & 36 & $3 *$ & -64.5 & 60 & 2 & -74.1 \\
\hline 6 & 1 & -46.0 & 38 & 2 & -64.4 & 61 & 2 & 10.0 \\
\hline 7 & 1 & -51.1 & 40 & 2 & -63.8 & 62 & 4 & 9.2 \\
\hline 8 & 1 & -69.1 & 41 & 1 & -72.3 & 63 & 3 & 8.1 \\
\hline 9 & 1 & -76.0 & 42 & 1 & -79.6 & 64 & 3 & -70.1 \\
\hline 10 & 1 & -80.9 & 43 & $3 *$ & -60.5 & 65 & 1 & -29.2 \\
\hline 11 & 1 & -79.0 & 44 & 1 & -63.1 & 66 & 2 & -75.8 \\
\hline 13 & 1 & -80.3 & 45 & $1 *$ & -48.4 & 68 & 2 & -73.0 \\
\hline 14 & 1 & -39.0 & 46 & 2 & -57.9 & 69 & 1 & -81.6 \\
\hline 16 & 1 & -74.9 & 47 & 1 & -55.3 & 70 & 3 & -79.3 \\
\hline 17 & $2^{*}$ & -64.6 & 48 & 1 & -45.4 & 71 & 2 & -69.6 \\
\hline 18 & 3 & -53.6 & 49 & 2 & -57.6 & 72 & 1 & -64.8 \\
\hline 19 & 2 & -68.7 & 50 & 2 & -77.3 & 73 & 3 & -64.5 \\
\hline 20 & $1 *$ & -56.6 & 52 & 4 & -80.8 & 74 & 1 & -78.2 \\
\hline 21 & 2 & -68.0 & 53 & $1 *$ & -85.9 & 76 & $3 *$ & -67.6 \\
\hline 21 & $3 *$ & -50.1 & 54 & 2 & -77.7 & 78 & 2 & -59.3 \\
\hline 23 & 2 & -44.4 & & & & 79 & 4 & -59.8 \\
\hline 24 & 2 & -39.7 & & & & 80 & 1 & -68.1 \\
\hline 25 & 3 & -61.8 & & & & 82 & 3 & -68.0 \\
\hline 26 & 2 & -60.0 & & & & 83 & 1 & -66.3 \\
\hline 27 & 2 & -62.8 & & & & 84 & 6 & -73.5 \\
\hline 28 & $1 *$ & -62.3 & & & & 85 & 2 & -79.1 \\
\hline 30 & 1 & -63.6 & & & & 86 & 2 & -77.0 \\
\hline 31 & 2 & -61.8 & & & & 87 & 4 & -50.4 \\
\hline 32 & 2 & -46.9 & & & & 88 & 1 & -76.4 \\
\hline \multirow[t]{4}{*}{33} & 2 & -38.5 & & & & 89 & 2 & -67.9 \\
\hline & & & & & & 90 & 2 & -77.6 \\
\hline & & & & & & 91 & 2 & -74.9 \\
\hline & & & & & & 92 & 2 & -74.9 \\
\hline
\end{tabular}

Notes: $\mathrm{N}=$ number of samples used for mean calculation. $\mathrm{Ch}$ inc $=$ mean characteristic inclination. ${ }^{*}=$ one of the values used in the mean is the inclination of a thermally remagnetized sample of the underlying flow.

could not use sophisticated statistical methods of averaging. Instead, simple arithmetic means of inclinations were calculated for each unit (Table 3).

\section{DISCUSSION}

\section{Short Event or Artifact?}

Shore-based AF demagnetization results obtained for samples from Units 61 through 63 and 65 confirm shipboard measurements. Furthermore, thermomagnetic curves obtained from the flows with shallow inclinations indicate the same magnetic mineralogy as other flows with steep negative inclinations. As expected, the main magnetic carrier is magnetite, with low or no titanium substitution. We can also conclude from the data in Table 1 that the susceptibilities, intensities, and Koenigsberger (1938) ratios in these particular flows are similar to those from other flows. Consequently, we think that flows 61-63 (and probably up to flow 65, which is characterized by a shallow negative inclination) have recorded a short excursion or normal event, called a cryptochron by Cande and Kent (1992, 1995).

If we consider the age of the volcanic sequence inferred from the oceanic magnetic anomaly (24r or older; see Larsen, Saunders, Clift, et al., 1994), we are not surprised to find a cryptochron, because Cande and Kent $(1992,1995)$ inferred that 11 cryptochrons occur within C24r, five within C25r, and seven within C26r. Chron C24r lasts 2.557 m.y. (Cande and Kent, 1995); considering 10 k.y. as a reasonable duration for a cryptochron, the geomagnetic field should be transitional for about 0.1 m.y. in 2.5 m.y. and should be recorded on average by one out of 25 flows. We drilled 92 flows and sampled 68; thus, it is to be expected that three or four flows show transitional directions if the sequence represents $\mathrm{C} 24 \mathrm{r}$. Similar frequency of appearance would be expected from $\mathrm{C} 25 \mathrm{r}$ and $\mathrm{C} 26 \mathrm{r}$. 


\section{Duration and Dating}

In a paleomagnetic study of a volcanic province, one must first demonstrate that paleosecular variation (PSV) has been sufficiently sampled. Adequate sampling of the PSV is an essential condition for the mean paleomagnetic direction to be considered representative of the past dipole field. Thus, the dispersion of virtual geomagnetic poles (VGP), the characteristic parameter typically used to estimate PSV, should be determined. For rotary cored material, such as volcanic rocks, the declination is unfortunately lost (see above). Consequently, the VGP cannot be calculated completely. As observed by Arason (1991), in the case of inclination-only data sets with steep inclinations and large scatter of magnetic directions, it becomes impossible to extract information concerning both inclination and scatter. Our results represent exactly such a case and prevent any obvious interpretation of inclination. Consequently, we use only polarity results to constrain the duration of SDRS volcanism.

The main conclusion about polarity obtained in this study is that the entire volcanic sequence is reversely magnetized, except flows 34 and 61-65, which probably recorded two cryptochrons (see above). Considering the lack of any noteworthy cessation of eruption activity, which would be indicated by interstratified sediments, we can infer that the extrusion of the Lower and Middle Series at Site 917 occurred during a single reversed polarity period. The Upper Series is separated from the lowerlying lavas and could belong to the same magnetic chron or a younger one. In the latter hypothesis, the time jump over a complete normal magnetic chron implies that the cessation of volcanic activity between Middle and Upper Series is at least $360 \mathrm{k} . \mathrm{y}$. long (duration of the shorter normal chron of the considered period: C26n).

The oceanic magnetic anomalies locate the SDRS in Anomaly 24r, between 53.3 and 55.9 Ma (Cande and Kent, 1995). Ar/Ar dating, however, places the SDRS around 60-63 Ma (Sinton and Duncan, this volume), which corresponds to Chrons C27r and C26r (Cande and Kent, 1995). In this period, reversed chrons last between 1.2 and 3.0 m.y. (Cande and Kent, 1995), which might be considered as an upper limit for the duration of the Lower and Middle Series at Site 917.

On the other hand, if we accept the hypothesis that a cryptochron is recorded by Units 61 to 65 (5 flows), considering $10 \mathrm{k} . \mathrm{y}$. as a reasonable duration for a cryptochron (Cande and Kent, 1992), and if we assume a relatively constant extrusion rate (i.e., about 5 flows per 10 k.y.), the complete Lower Series (35 units) should represent less than 70 k.y. The same rough calculation for the Middle Series gives about $240 \mathrm{k} . \mathrm{y}$. with one transitional direction over 24 units. These duration estimates for Lower and Middle Series (respectively 70 and 240 k.y.) are also consistent with the duration of intervals between two cryptochrons in C26r, C25r, and C24r, mainly about $250 \mathrm{k} . y$. and spreading out from 100 to 540 k.y. A similar estimate for the Upper Series is impossible because the lack of transitional directions can be interpreted as reflecting either a low extrusion rate (much less than one flow per 10 k.y.), which is insufficient to record a cryptochron and implies a long extrusion period (much more than 330 k.y. for 33 units), or a high extrusion rate during a short period, between two cryptochrons (possibly less than 100 k.y.).

Furthermore, the probable presence of two cryptochrons would favor the hypothesis that the SDRS is located in Anomalies 24r (11 cryptochrons) or $26 \mathrm{r}$ (seven cryptochrons), rather than in $27 \mathrm{r}$ (no recognized cryptochron). An intermediate solution, Anomaly 25r, would, on one hand, minimize the gap between SDRS extrusion and the first recognized oceanic anomaly (about $3 \mathrm{~m} . \mathrm{y}$.) and, on the other hand, suggest a possible argon excess in $\mathrm{Ar} / \mathrm{Ar}$ dating (between 3 and $6 \mathrm{~m} . \mathrm{y}$.). From the point of view of a paleomagnetist, the latter hypothesis is also possible, reflecting five recognized cryptochrons and a maximum duration of about 1.2 m.y.

\section{CONCLUSION}

The paleomagnetic study of the volcanic sequence cored in Hole 917A provides important constraints on its possible age but no clues on the tectonic history of the SDRS. The reversed magnetization of the entire volcanic sequence and the observation of at least two short geomagnetic events (one in the Lower Series and one in the Middle Series) are consistent with the proposed ages of SDRS in Anomalies $24 \mathrm{r}, 25 \mathrm{r}$, or $26 \mathrm{r}$, which contain 11 , five, and seven cryptochrons, and which last 2.6, 1.2, and 3.0 m.y., respectively.

The interstratified sediment level that separates Upper Series from lowerlying lavas indicates a cessation of volcanic activity. The reversed polarity of the complete sequence of Hole 917A can consequently be interpreted by volcanism during a single reversed chron or during two successive reversed chrons. The latter hypothesis implies a cessation of activity during at least 360 k.y., which makes longer the duration of the Hole 917A sequence.

The duration of the Upper Series is only constrained by magnetostratigraphy: its reversed polarity without apparent cessation of activity implies that volcanism of the Upper Series took place during one reversed chron. In contrast, the good recording of two cryptochrons in the Lower and Middle Series shows a short period of high volcanic activity, during about 300 k.y.

\section{REFERENCES}

Arason, P., 1991. Paleomagnetic inclination shallowing in deep-sea sediments [Ph.D. thesis]. Oregon State Univ., Corvallis, Oregon.

Cande, S.C., and Kent, D.V., 1992. A new geomagnetic polarity time scale for the Late Cretaceous and Cenozoic. J. Geophys. Res., 97:1391713951.

- 1995. Revised calibration of the geomagnetic polarity timescale for the Late Cretaceous and Cenozoic. J. Geophys. Res., 100:6093-6095.

Kirschvink, J.L., 1980. The least-squares line and plane and the analysis of palaeomagnetic data. Geophys. J. R. Astron. Soc., 62:699-718.

Koenigsberger, J.G., 1938. Natural residual magnetism of eruptive rocks. Terr. Magn. Atmos. Electr., 43:119-127.

Larsen, H.C., Saunders, A.D., Clift, P.D., et al., 1994. Proc. ODP, Init. Repts., 152: College Station, TX (Ocean Drilling Program).

Shipboard Scientific Party, 1994. Site 917. In Larsen, H.C., Saunders, A.D., Clift, P.D., et al., Proc. ODP, Init. Repts., 152: College Station, TX (Ocean Drilling Program), 107-158.

Thellier, E., and Thellier, O., 1959. Sur l'intensité du champ magnétique terreste dans le passé historique et geologique. Ann. Geophys., 15:285-375.

Zijderveld, J.D.A., 1967. AC demagnetization of rocks: analysis of results. In Collinson, D.W., Creer, K.M., and Runcorn, S.K. (Eds.), Methods in Palaeomagnetism: New York (Elsevier), 254-286.

Date of initial receipt: 2 November 1995

Date of acceptance: 11 November 1996 Ms 152SR-223 